\title{
Comprehensive Study of Facial Action Coding
}

\author{
Ali Alomari \\ School of Arts and Science \\ Clark Atlanta University, Atlanta, GA, USA
}

\begin{abstract}
Facial Action Coding (FAC) is expected to apply the whole world. The solicitations are not controlled to media communications. FAC is a testing difficulty in computer vision and remains alarm security case, and a novel strategy for program FAC is guided to bargain nearby the issue. The principle trial here, head-case and non-unbending outward appearance conformities because of alterations invited by the brutal face decoupling to present as they are coupled to the non-direct pictures. One more trial is the way to proficiently request to empower affiliation to contemplate expression (or different facial components) is to misuse the data. Facial Expression(FE) picture succession is fleeting territory spatial region information directly rise, however moreover the advance is not known. Information considering the increase of appearance together nearby the photo participation information can more improve the presentation of acknowledgment. However, the active information supplied is practical, there how to capture this information dependably and powerfully concerning facing challenges. For instance, an FE arrangement typically, one or additional of the principle times of development and counterbalance beat. Temporary information and preparing with a specific end goal to capture and question transient groupings of identical information, to make the correspondence in the midst of different worldly periods request to be built up. Press can be encoded. In this work, another dynamic FE, hereditary and neural organize based way utilizing the half, and half method are made.
\end{abstract}

\section{General Terms}

Facial Action Coding

\section{Keywords}

Facial Action Coding, Facial Expression Recognition (FER), and Testing Faces.

\section{INTRODUCTION}

There are various troubles in outward appearance acknowledgment (FER) because of the variety of outward appearance over the human populace and to the setting subordinate variety even for a similar person. Indeed, even we people may commit errors [1]. Then again, FER by PC is precious in numerous applications, for example, human conduct understanding and human-PC interface. A program FER framework needs to take care of the accompanying issues: discovery and area of confronts in a messed scene, facial component extraction, and outward appearance arrangement. Face numerous scientists have considered recognition, and it appears that best frameworks depend on neural systems $[2,3]$. Once a face is identified in the picture, the relating locale is separated, and is standardized to have a similar size (for instance, the same remove between two eyes) and a similar dim level. In this paper, we don't address the face recognition issue.
Facial element extraction endeavors to locate the most proper representation of the face pictures for acknowledgment. There are mostly two methodologies: all-encompassing layout coordinating frameworks and geometric component based frameworks [4]. In all-encompassing structures, a design can be a pixel picture or a highlight vector got in the wake of handling the face picture overall. In the last mentioned, chief part examination and multilayer neural systems are broadly used to get a low-dimensional representation. In geometric element based frameworks, significant face parts or potentially include focuses are identified in the pictures. The separations between highlight focuses and the relative sizes of the major confront segments are figured to frame a component vector. The component focuses can likewise shape a geometric chart representation of the appearances. Include based methods are normally computationally more costly than layout based systems, yet are more powerful to variety in scale, estimate, head introduction, and area of the face in a picture. The work to be depicted in this paper is, to some degree, a crossover approach. We first find an arrangement of highlight focuses, and afterward, remove an arrangement of Gabor wavelet coefficients at every point through picture convolution. Contrasted and confront acknowledgment, there is a little measure of work on outward appearance acknowledgment. The primary classification of past work utilizes picture groupings. Suwa et al. [5] did a preliminary examination of outward appearances by following the movement of twenty distinguished spots. Mase [6] utilizes the methods and differences of optical stream information at uniformly separated little pieces. Yacoob and Davis [7] utilize the between causing movement of edges separated in the zone of the mouth, eyes, and eyebrows. Bartlett et al. [8] utilize the blend of the optical stream, and intermediate segments got from picture contrasts. Essa and Pentland [9] fabricates an parametric element show by following facial movement after some time, which can then be utilized for breaking down facial expressions. The second classification of past work tries to group outward appearances from static pictures. Turk and Pentland [10] speak to face pictures by eigenfaces through direct middle segment investigation. Padgett and Cottrell [11] utilize an approach like eigenfaces however with seven-pixel hinders from highlight locales (both eyes and mouth). Cottrell and Metcalfe [12] employ comprehensive representations given key parts, extricated by nourish forward systems. Rahardja et al. [13] likewise utilize comprehensive representations with neural systems. However the pictures are spoken to in a pyramid structure. Lanitis et al. [14] utilize parameterized deformable layouts (adaptable models) which consider both varieties fit as a fiddle and deep level appearance. 


\section{STEPS TO PROCESS FAC}

The following are the two method steps FAC processes

2.1 Training Result

2.2 Testing Result as shown in Figure 1 and 2.

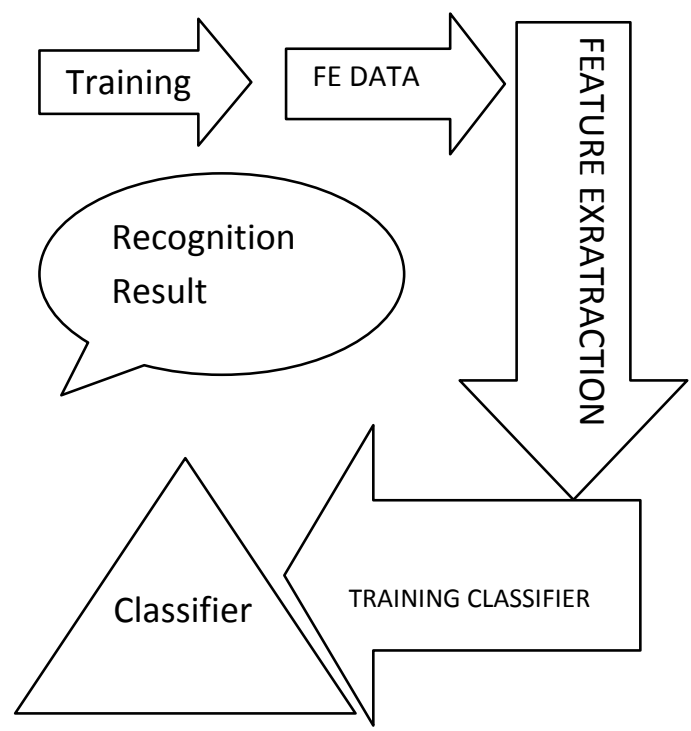

Figure 1: Training Face Recognition Result

\subsection{Testing}

2.4 Classification

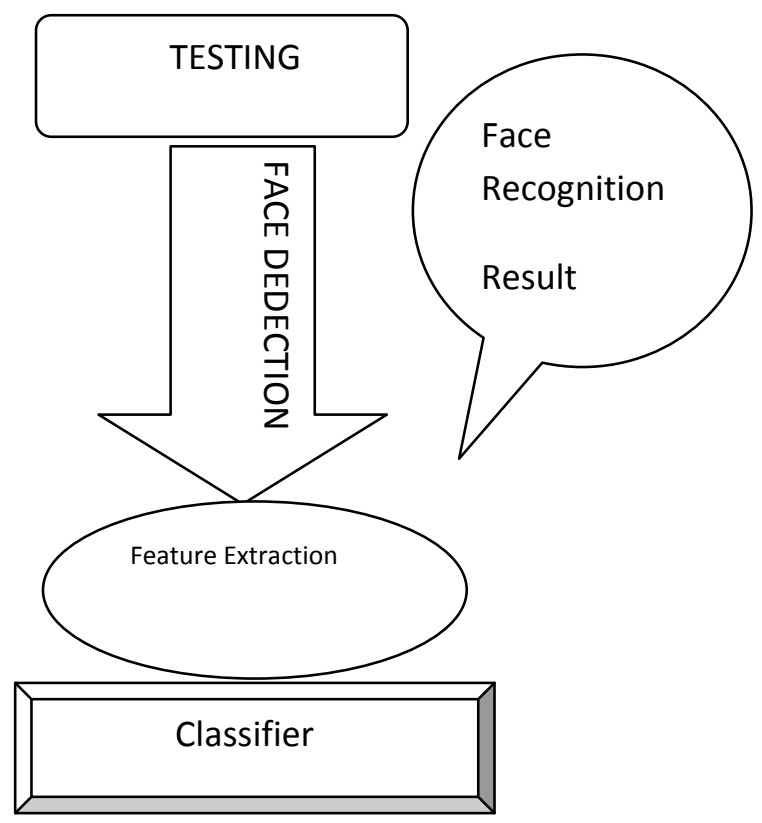

Figure 2: Testing Face Recognition Result

\section{DIFFICULTIES IN RESEARCH}

There are endless angles to be educated: the giant tedious and complex that incalculable models include focuses or particular traverses that are set apart by hand. Expression pictures, alterations in the statement of both facial credit thus now and then include information evacuated components are regularly a mix of conformities in the expression and uniqueness qualities. Unique requests and highlight extraction (Figure 2) in the result at different forces, yet a little review on the face close to light. For example, the eyes, temple, vague as key ranges, highlight extraction will adjust, also, hinder nearby the FEC.

\section{DEVELOPMENT TREND OFFER}

Development Trend of FE Recognition [23]

1) Measurement of the Distance

Expression changing pictures include information perceiving information and expression. In this way, a skilled extent of articulation of every two pictures agreed and exact significance needs more find.

2) Feature Extraction of Micro-Expression Small Scale Expression short discourse perpetual no additional than $1 / 4 \mathrm{~S}$ notices to the program distinguishing proof of the challenges in this brief period and is not easy to be accepted, but rather it can express non-subjective sentiments.

3) The Psychological Study FE mirrors the soul of the individual, mental alterations moreover mimic changes in the face. Passionate alterations, and close by the vision of how human brain science of components expelled utilizing different parameters to inspect a convoluted enthusiastic perspective, however, beneficial trial.

\section{4) Multi-ethnic}

it is significant to the expression acknowledgment and will turn into a hotspot to know the relations and distinctive between various ethnic gatherings' facial elements.

\section{5) Three-Dimensional Face Recognition}

3D confront acknowledgment, then the two-dimensional light picture acknowledgment, confront demeanor, is helpless against the antagonistic impacts of the material secured on the face. 3D database creation $\mathrm{FE}$ acknowledgment will give finish data. It is essential that somewhere in the range of 3D database is introduced.

6) Face Recognition Based on Multi-include Fusion Multihighlight acknowledgment framework is utilized as a part of a request to enhance include extraction and enhance the strength. Step by step instructions to locate a solid and exact calculation for nearby element extraction and reconciliation is a vital research bearing.

7) The Integration of FE Recognition with other Biometric Identification Technologies

Some human organic qualities are distinctions and won't effortlessly change, for example, facial components, eye trademark (retina, iris), and unique finger impression. It ought to limitlessly enhance distinguish framework execution to combine confront acknowledgment and other biometric recognizable proof advancements.

\section{POTENTIAL APPLICATION}

The potential advantages from endeavors to computerize the investigation of outward appearances differ and various what's more, traverse fields as assorted as psychological sciences, pharmaceutical, correspondence, training, and security [15]. With regards to software engineering and processing advancements, outward appearances give an approach to impart fundamental data about requirements and requests to the machine. Too, certain facial signs (e.g., a wink) can be related to specific orders (e.g., a mouse click) advertising a different option to traditional console and mouse orders. The 
human ability to "listen" in big situations by a method for lip perusing is the reason for bimodal (varying media) discourse handling. To make a convincing talking head (symbol) speaking to a genuine individual, perceiving the individual's facial signs and making the symbol react to those utilizing integrated discourse and outward appearances is vital. Consolidating outward appearance spotting with outward appearance elucidation as far as names prefer 'did not get it", 'dissent", "absentminded", and "supports" could be utilized as an apparatus for checking human responses amid video conferences, online addresses, and mechanized coaching sessions. The concentration of the moderately, as of late started inquire about region of full of feeling processing lies on detecting, identifying and translating human full of feeling states, (for example, satisfied, aggravated, befuddled, and so forth.) what's more, formulating fitting means for taking care of this full of feeling data so as to improve current. The unsaid suspicion is that much of the time human-machine communication could be made strides by the presentation of machines that can adjust to their clients and how they feel

\section{RELATED WORK VARIOUS FACE TECHNIQUES}

1. Eigenfaces for Recognition: Holistic approach utilizing pixel power for highlight extraction then dimensionality diminishment with Principal part investigation and arranged to utilize closest neighbor classifier [16]

2. ICA-based component analysis: ICA is superior to PCA catches both second and higher request insights and ventures the information onto the premise vectors that are measurably independent [17].

3. Two-dimensional PCA another way to deal with appearance based confront representation and acknowledgment: This strategy utilizes specifically $2 \mathrm{D}$ picture grids as opposed to 1D vectors for covariance grid estimation as in PCA, it is numerically modest and basic, more appropriate for little specimen estimate approaches. [18]

4. Confront acknowledgment utilizing Local parallel examples: LBP elements are powerful to low determination pictures.

5. Low-Resolution Single Neural Network-Based Face Acknowledgment: It utilizes the single neural system as a classifier, which produces direct approach towards confronting acknowledgment. It applies for bi-cubic addition work on adjusted picture to get resized picture which is low resolution picture, therefore, giving quicker preparing to prepare and testing.[18]

6. Segment based face acknowledgment with 3D Morphable Models: It is vigorous, exact, and effortlessly trainable face acknowledgment framework. The part based framework reliably beat worldwide face acknowledgment frameworks in which classification depended on overall confront pattern.[19]
7. Acknowledgment of outward appearances utilizing Gabor wavelets Furthermore, learning vector quantization: It is watched that LVQ based component grouping procedure proposed in this ponder performs better in perceiving dread expressions than multilayer perceptron (MLP) based arrangement technique.[20][21][22].

\section{FACE RECOGNITION}

A few novel approaches to fathom every change recorded above have been proposed. For instance, a standard face design for layout coordinating strategies for processing the relationship of an info picture is utilized for the face location and restriction. Office irreversible vision eyes, mouth, ears, nose, and so forth nearness Eigen confront neural system based techniques and hypothetical methodologies with the face discovery data that is utilized for identification are utilized for accommodation. Nonetheless, to completely execute the techniques is still a major test. Luckily, the pictures utilized as a part of the venture have some level of consistency; the location calculation is straightforward: First, every one of the appearances is steep and the frontal view; Second, they are nearly the same lighting up condition. The venture, for the most part, shading partition, picture division and layout coordinating strategies in light of face location innovation presents. body.

\section{FACE RECOGNITION PROCEDURE}

The proposed strategy includes two stages - encoding and interpreting. There are five phases in the encoding stage, the key facial regions including extraction, wavelet change, the now - uniform quantization and pick the level of deterioration, versatile number-crunching encoding, and field-based encoding progressive tree. To clarify the gauge strategy, wavelet change; Non - uniform quantization and pick the level of deterioration; Area - based encoding and versatile number juggling encoding progressive tree countenances are set as picture pressure module. Key regions of the face extraction arrange, the projection of the picture by utilizing data, the key regions of the face are separated. Furthermore, the first picture is separated into key regions and foundations.

\section{CONCLUSIONS}

As of late, the dynamic FE acknowledgment has turned into a new research subject and got consideration increasingly. The issue is unique about the acknowledgment of static pictures, dynamic FE acknowledgment of the physical question of a picture succession of a subject caught amid the procedure of FE Sort surmising. FE picture arrangement fleeting area spatial area data just appearance, additionally the Improvement is not known. Data on the improvement of expression together with the picture nearness data can additionally upgrade the execution of acknowledgment. Nonetheless, the dynamic data gave is valuable, there how to catch this data dependably and vigorously about confronting challenges. The Eigenface of the procedures utilized as a part of the trial framework, and after that sections, the head, eyes, lips and button identifies facial Picture is utilizing. Sobel administrator is connected to the picture to get the Double histogram. At that point utilize the territory as the component vector, framework preparing and data from the database by taking Both live cameras is utilized. The last stride was to test pictures utilizing two methodologies; Genetic calculations and neural system. 


\section{ACKNOWLEDGMENT}

This research supported by Saudi Arabian Cultural Mission. This support is entirely thankful.

\section{REFERENCES}

[1] P. Ekman and W. Friesen. Unmasking the Face: A guide to recognizing emotions from facial expressions. Consulting Psychologists Press, Palo Alto, CA, 1975.

[2] K.-K. Sung and T. Poggio. Example-based learning for view-based human face detection. Technical Report A.I. Memo 1521, CBCL Paper 112, MIT, Dec. 1994.

[3] H. Rowley, S. Baluja, and T. Kanade. Human face detection in visual scenes. Technical Report CMU-CS95-158R, School of Computer Science, Carnegie Mellon University, Nov. 1995.

[4] R. Chellappa, C. Wilson, and S. Sirohey. Human and machine recognition of faces: A survey. Proceedings of the IEEE, 83(5):705-740, May 1995.

[5] M. Suma, N. Sugie, and K. Fujimora. A preliminary note on pattern recognition of human emotional expression. In Proceedings of the 4th International Joint Conference on Pattern Recognition pages 408-410, 1978.

[6] K. Mase. Recognition of facial expression from optical flow. IEICE Transactions E, 74(10):3473-3483, 1991.

[7] Y. Yacoob and L. Davis. Recognizing facial expressions by spatiotemporal analysis. In Proceedings of the International Conference on Pattern Recognition, volume 1, pages 747-749, Jerusalem, Israel, Oct. 1994. Computer Society Press.

[8] M. Bartlett, P. Viola, T.Sejnowski, L. Larsen, J. Hager, and P. Ekman. Classifying facial action. In D. Touretzky, M. Mozer, and M. Hasselmo, editors, Advances in Neural Information Processing Systems 8. MIT Press, Cambridge, MA, 1996.

[9] I. Essa and A. Pentland. Coding, analysis, interpretation, and recognition of facial expressions. IEEE Transactions on Pattern Analysis and Machine Intelligence, 19(7):757-763, July 1997.

[10] M. Turk and A. Pentland. Eigenfaces for recognition. J. of Cognitive Neuroscience, 3(1):71-86, Mar. 1991.

[11] C. Padgett and G. Cottrell. Identifying emotion in static images. In Proceedings of the 2nd Joint Symposium on Neural Computation, volume 5, pages 91-101, La Jolla, CA, 1997.

[12] G. Cottrell and J. Metcalfe. Face, gender and emotion recognition using holons. In D. Touretzky, editor, Advances in Neural Information Processing Systems 3, pages 564-571. Morgan and Kaufman, San Mateo, 1991.
[13] A. Rahardja, A. Sowmya, and W. Wilson. A neural network approach to component versus holistic recognition of facial expressions in images. In Intelligent Robots and Computer Vision X: Algorithms and Techniques, Volume 1607 of SPIE Proc., pages 62-70, 1991.

[14] A. Lanitis, C. Taylor, and T. Cootes. Automatic interpretation and coding of face images using flexible models. IEEE Transactions on Pattern Analysis and Machine Intelligence, 19(7):743-756, July 1997.

[15] Ekman, P., Huang, T.S., Sejnowski, T.J., Hager, J.C. (eds.): NSF Understanding the Face. A Human Face eStore, Salt Lake City, USA, (see Library) (1992).

[16] M. Turk and A. Pentland, Face Recognition Using Eigenfaces, Proc. IEEE Conf. on Computer Vision and Pattern Recognition, pp. 586-591, 1991.

[17] M.S. Bartlett, J.R. Movellan and T.J. Sejnowski, Face recognition by independent component analysis, IEEE Transaction on Neural Networks, Vol 13,pp. 14501464,2002 .

[18] Z. Jahan, M.Y. Javed and Q. Usman, Low-resolution single Neural Network based Face Recognition, Proceedings of the Fourth International Conference on Computer Vision, Image and Signal Processing, Vol. 22, pp. 189193, 2007.

[19] TimoAhonen, AbdenourHadid, and MattiPietikainen Face Description with Local Binary Patterns: Application to Face Recognition in IEEE Transactions on Pattern Analysis and Machine Intelligence, Vol. 28, No. 12, December 2006.

[20] S. Bashyal and G.K. Venayagamoorthy, Recognition of Facial expres-sions using Gabor Wavelets and Learning Vector Quantization, Engineer-ing Applications of Artificial Intelligence, Vol. 21, pp. 10561064, 2008.

[21] De Stefano, C., Sansone, C. and Vento M., Comparing generalization and recognition capability of Learning Vector Quantization and Multilayer Perceptron Architectures, Proceedings of the 9th Scandinavian Confer-ence on Image Analysis, pp. 11231130, June 1995.

[22] Divya Bhatnagar, Drashti Pathak, Garima Saini, Amit Kumar Gautam and Vijai Singh. Article: A Review: Analysis of Facial Micro-Expressions. IJCA Proceedings on Recent Trends in Future Prospective in Engineering and Management Technology RTFEM 2016(2):1-3, July 2016.

[23]Anisha Shadi and Anil Khandelwal. Multi-scale and Multi-orientation Face Recognition using Voting based Extreme Learning Machine. International Journal of Computer Applications 152(1):52-56, October 2016. 